TITLE:

\title{
Path integral evaluation of the quantum instanton rate constant for proton transfer in a polar solvent
}

\author{
AUTHOR(S): \\ Yamamoto, T; Miller, WH
}

\section{CITATION:}

Yamamoto, $T$... [et al]. Path integral evaluation of the quantum instanton rate constant for proton transfer in a polar solvent. JOURNAL OF CHEMICAL PHYSICS 2005, 122(4): 044106.

\section{ISSUE DATE:}

2005-01-22

URL:

http://hdl.handle.net/2433/50583

\section{RIGHT:}

Copyright 2005 American Institute of Physics. This article may be downloaded for personal use only. Any other use requires prior permission of the author and the American Institute of Physics. 


\title{
Path integral evaluation of the quantum instanton rate constant for proton transfer in a polar solvent
}

\author{
Takeshi Yamamoto ${ }^{\text {a) }}$ and William H. Miller ${ }^{\text {b) }}$ \\ Department of Chemistry and Kenneth S. Pitzer Center for Theoretical Chemistry, University of California, \\ Chemical Sciences Division, Lawrence Berkeley National Laboratory, Berkeley, California 94720
}

(Received 10 September 2004; accepted 22 October 2004; published online 5 January 2005)

\begin{abstract}
The quantum instanton approximation for thermal rate constants, a type of quantum transition state theory (QTST), is applied to a model proton transfer reaction in liquid methyl chloride developed by Azzouz and Borgis. Monte Carlo path integral methods are used to carry out the calculations, and two other closely related QTST's, namely, the centroid-density and Hansen-Andersen QTST, are also evaluated for comparison using the present path integral approach. A technique is then introduced that calculates the kinetic isotope effect directly via thermodynamic integration of the rate with respect to hydrogen mass, which has the practical advantage of avoiding costly evaluation of the activation free energy. The present application to the Azzouz-Borgis problem shows that the above three types of QTST provide very similar results for the rate, within 30\% of each other, which is nontrivial considering the totally different derivations of these QTSTs; the latter rates are also in reasonable agreement with some other previous results (e.g., obtained via molecular dynamics with quantum transitions), within a factor of $\sim 2(7)$ for the $H(D)$ transfer, thus significantly diminishing the possible range of the exact rates. In addition, it is revealed that a small but nonnegligible inconsistency exists in the parametrization of the Azzouz-Borgis model employed in previous studies, which resulted in the large apparent discrepancy in the calculated rates. (C) 2005 American Institute of Physics. [DOI: 10.1063/1.1832598]
\end{abstract}

\section{INTRODUCTION}

As is well appreciated, the reaction rate of hydrogen transfer is sometimes significantly impacted by nuclear quantum effects of the transferring hydrogen. This is to be expected for reactions at low temperature or with a high activation barrier, but it is also known ${ }^{1,2}$ that for some enzymatic reactions the hydrogen transfer proceeds via tunneling even under physiological conditions with the help of thermal protein motions (called "promoting" or "gating" vibrations) that directly modulate the donor-acceptor distance. A variety of theoretical approaches have been used to investigate such an involved quantum rate process by computer simulation, ${ }^{3-8}$ among which of our concern in this paper (i.e., related to Feynman's path integral in imaginary time $)^{9,10}$ is the centroid-density quantum transition state theory (QTST). ${ }^{11-14}$ This method adopts a rate expression identical to the classical TST, but defines the activation free energy in terms of the centroid of the imaginary-time path in order to account for the quantum delocalization of the hydrogen. Another theoretical method of concern is the QTST developed by Hansen and Andersen, ${ }^{15}$ which is somewhat more "first-principles" and considers a direct short-time approximation to the rigorous quantum mechanical flux-flux correlation function. ${ }^{16}$ The application of the Hansen-Andersen method, however, has been limited to simple model systems

\footnotetext{
a) Present address: Department of Chemistry, Graduate School of Science, Kyoto University, Kyoto 606-8502, Japan; Electronic mail: yamamoto@kuchem.kyoto-u.ac.jp

b)Electronic mail: miller@cchem.berkeley.edu
}

to date, ${ }^{15,17}$ because it was not obvious how to evaluate the latter theory efficiently using path integral techniques rather than basis set methods.

A recent paper $^{18}$ has introduced a different QTST that was motivated by an earlier semiclassical (SC) transition state theory ${ }^{19}$ (also known as the "instanton" model) ${ }^{20}$ and is thus referred to as the quantum instanton (QI) approximation. The similarity between the quantum and SC instanton theories lies in the fact that steepest descent approximations are used to evaluate the relevant integrals in the quantum mechanical rate expression, while the critical difference is that the Boltzmann operators involved in the QI rate expression are evaluated fully quantum mechanically rather than within an SC approximation. (Here we recall that the steepest descent evaluation of the Boltzmann operator leads to the well-known classical periodic orbit on an "upside-down" potential energy surface. $)^{19}$ The QI theory thus incorporates all the tunneling contributions correctly and is expected to overcome the quantitative deficiency of the SC instanton model. ${ }^{21}$ Indeed, several test applications have shown the QI theory to give accurate quantum rates over a wide temperature range, from the "deep" tunneling regime at low temperature to the regime of over-barrier dynamics at high temperature. ${ }^{18,22-26}$ A practical path integral scheme has also been developed to evaluate the QI rate for more complex chemical reactions, ${ }^{22}$ with which the QI theory was applied to the gas-phase $\mathrm{H}+\mathrm{CH}_{4} \rightarrow \mathrm{H}_{2}+\mathrm{CH}_{3}$ reaction with all the methane hydrogens treated symmetrically in full Cartesian space. $^{23}$

The purpose of this paper is thus to explore the applica- 
bility of the QI theory to a realistic chemical reaction in a condensed phase. To this end we study a model proton transfer reaction in solution developed by Azzouz and Borgis, ${ }^{27}$

$$
A H+B \rightarrow A^{-}+H^{+} B,
$$

where $A, H$, and $B$ constitute a typical phenol-amine $\mathrm{H}$-bonding complex dissolved in liquid methyl chloride. The reason for choosing this system is twofold. First, the Azzouz-Borgis model embodies several important aspects of condensed-phase proton transfer reactions (e.g., donoracceptor modes that directly modulate the potential barrier and a polar solvent that qualitatively changes the free energy profile via electrostatic interactions). Second, although a variety of theoretical approaches have been applied to this model, ${ }^{27-31}$ the calculated rates do not agree well and thus the exact quantum rate is still unknown. More specifically, Azzouz and Borgis investigated the above model using a semiclassical curve-crossing TST and the centroid-density QTST. $^{27}$ Subsequently it was studied by other groups using molecular dynamics with quantum transitions (MDQT), ${ }^{28,29}$ quantum Kramers approaches, ${ }^{30}$ and variational transition state theory with multidimensional tunneling corrections (VTST/MT). ${ }^{31}$ The calculated rates differ by more than two orders of magnitude, implying the difficulty of obtaining quantitatively accurate proton transfer rates in solution. The Azzouz-Borgis model thus represents a rather nontrivial application for the different QI theory.

The remainder of this paper is as follows. In Sec. II we first summarize the Azzouz-Borgis model and present the working expressions of the QI theory. We then describe the path integral framework for evaluating the QI rate and how it can be used also to deal with the Hansen-Andersen QTST. A technique is then introduced that directly calculates the kinetic isotope effect (KIE) via thermodynamic integration of the rate with respect to hydrogen mass. In Sec. III we discuss important features of the relevant free energy surfaces, examine the statistical convergence rate of path integral estimators, and compare the resulting QI rates with the previous results. In Sec. IV we summarize the main conclusions in this paper.

\section{METHODOLOGY}

\section{A. The Azzouz-Borgis model for proton transfer in polar solvent}

In this section we describe the key aspects of the Azzouz-Borgis model. ${ }^{27}$ We follow the specific version of the model given by Hammes-Schiffer and Tully ${ }^{28}$ because all the previous studies except for Ref. 27 have employed their parametrization. The present model deals with a phenolamine H-bonding complex (AHB) dissolved in liquid methyl chloride, where $A$ and $B$ are two Lennard-Jones (LJ) centers with masses corresponding to phenol $\left(M_{A}=93 \mathrm{amu}\right)$ and trimethylamine $\left(M_{B}=59 \mathrm{amu}\right)$, respectively, while the proton $H$ (with $m_{H}=1 \mathrm{amu}$ ) is constrained to move along the $A B$ axis, i.e., the complex is treated as a collinear triatomic molecule.
TABLE I. Parameters used in the gas-phase H-bonding potential $V_{\mathrm{HB}}\left(r, R_{A B}\right)$.

\begin{tabular}{lc}
\hline \hline \multicolumn{1}{c}{ Parameter } & Value \\
\hline$a\left(\AA^{-1}\right)$ & 11.2 \\
$b(\mathrm{kcal} / \mathrm{mol})$ & $7.1 \times 10^{13}$ \\
$d_{A}(\AA)$ & 0.95 \\
$d_{B}(\AA)$ & 0.97 \\
$D_{A}(\mathrm{kcal} / \mathrm{mol})$ & 110 \\
$n_{A}\left(\AA^{-1}\right)$ & 9.26 \\
$n_{B}\left(\AA^{-1}\right)$ & 11.42 \\
$c$ & 0.776 \\
\hline
\end{tabular}

We employ the following Cartesian form of the gasphase solute Hamiltonian in order to simplify the path integral calculation,

$$
H_{\mathrm{gas}}=\frac{1}{2} M_{A} \dot{\mathbf{R}}_{A}^{2}+\frac{1}{2} M_{B} \dot{\mathbf{R}}_{B}^{2}+\frac{1}{2} m_{H} \dot{r}^{2}+V_{H B}\left(r, R_{A B}\right),
$$

where $r=\left|\mathbf{R}_{A}-\mathbf{R}_{H}\right|$ and $R_{A B}=\left|\mathbf{R}_{A}-\mathbf{R}_{B}\right|$ with $\mathbf{R}_{\alpha}(\alpha$ $=A, H, B)$ being the Cartesian coordinates of site $\alpha$. Although the above form is not strictly identical to those used in previous work, their difference can be shown to be negligible due to the large disparity in masses (see Appendix A for details). The gas-phase H-bonding potential $V_{H B}$ in Eq. (2.1) is given by

$$
\begin{aligned}
V_{H B}\left(r, R_{A B}\right)= & b e^{-a R_{A B}}+D_{A}\left\{1-\exp \left[\frac{-n_{A}\left(r-d_{A}\right)^{2}}{2 r}\right]\right\} \\
& +c D_{A}\left\{1-\exp \left[\frac{-n_{B}\left(R_{A B}-r-d_{B}\right)^{2}}{2\left(R_{A B}-r\right)}\right]\right\},
\end{aligned}
$$

where the first term in the right-hand side represents the core repulsion between $A$ and $B$, while the remaining terms describe the chemical bonding of $H$ with $A$ and $B$. The parameters in Eq. (2.2) are listed in Table I. We depict the above gas-phase potential $V_{H B}$ in Fig. 1. It is seen from this figure that the present $V_{H B}$, which is based on the parametrization by Hammes-Schiffer and Tully, ${ }^{28}$ has a barrier height of $\sim 2 \mathrm{kcal} / \mathrm{mol}$ smaller than that of Azzouz and Borgis (Fig. 1 in Ref. 27). This difference has not been emphasized in previous work but is non-negligible in discussing the rate constants (see Sec. III).

The Azzouz-Borgis model treats the solvent methyl chloride molecule as a rigid, polar, nonpolarizable diatomic, where the methyl group is reduced to a united atom. The solvent-solvent interaction potential, $V_{\mathrm{SS}}$, is given by a TIPS (transferable intermolecular potential functions) form as follows:

$$
\begin{aligned}
V_{\mathrm{SS}}\left(\mathbf{R}_{S}\right)= & \frac{1}{2} \sum_{k \neq k^{\prime}} \sum_{\beta, \beta^{\prime}=\mathrm{Me}, \mathrm{Cl}}\left(\frac{q_{\beta} q_{\beta^{\prime}}}{\left|\mathbf{R}_{k}^{\beta}-\mathbf{R}_{k^{\prime}}^{\beta^{\prime}}\right|}+\frac{A_{\beta} A_{\beta^{\prime}}}{\left|\mathbf{R}_{k}^{\beta}-\mathbf{R}_{k^{\prime}}^{\beta^{\prime}}\right|^{12}}\right. \\
& \left.-\frac{C_{\beta} C_{\beta^{\prime}}}{\left|\mathbf{R}_{k}^{\beta}-\mathbf{R}_{k^{\prime}}^{\beta^{\prime}}\right|^{6}}\right)
\end{aligned}
$$

where $\mathbf{R}_{S}$ stands collectively for the solvent coordinates, while $\mathbf{R}_{k}^{\beta}$ is the Cartesian vector of site $\beta$ on the $k$ th solvent molecule. The TIPS parameters in Eq. (2.3) are listed in 


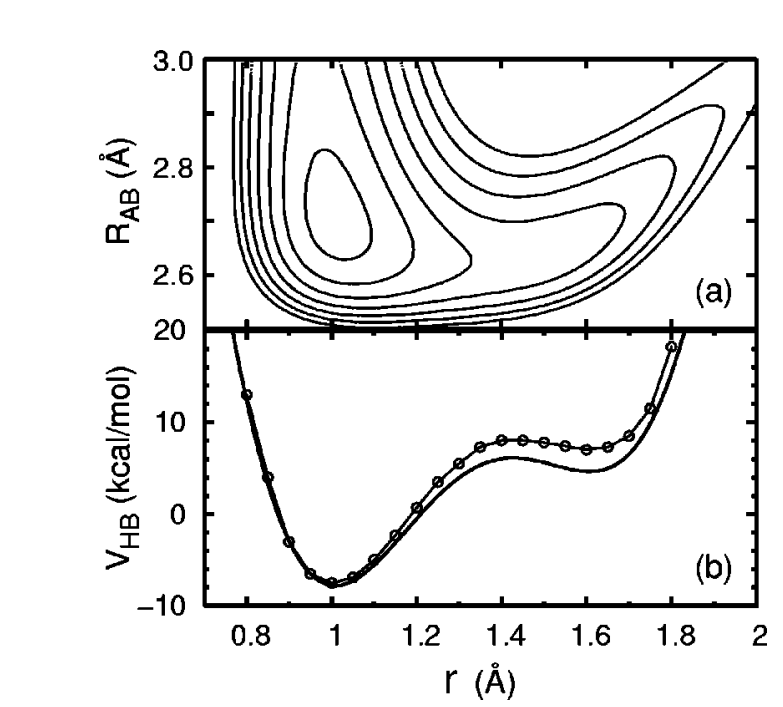

FIG. 1. Gas-phase H-bonding potential $V_{\mathrm{HB}}\left(r, R_{A B}\right)$ in Eq. (2.2). Zero of energy is taken to be the infinite separation limit $A H+B$ [thus a constant $c D_{A}$ is subtracted from $\left.V_{\mathrm{HB}}\left(r, R_{A B}\right)\right]$. (a) Contours are plotted for energies of $-6,-2, \ldots, 18 \mathrm{kcal} / \mathrm{mol}$; (b) section at $R_{A B}=2.7 \AA$. The present result is shown by solid line. The corresponding potential curve of Azzouz and Borgis is reproduced from Fig. 1 in Ref. 27 (line with circles).

Table II. The interaction potential between the H-bonding complex and the solvent molecules, $V_{\mathrm{CS}}$, is the sum of Coulomb and $\mathrm{LJ}$ interaction potentials,

$$
\begin{aligned}
V_{\mathrm{CS}}\left(r, \mathbf{R}_{A}, \mathbf{R}_{B}, \mathbf{R}_{S}\right)= & \sum_{\alpha=A, B, H} \sum_{k} \sum_{\beta=\mathrm{Me}, \mathrm{Cl}} \frac{q_{\alpha}(r) q_{\beta}}{\left|\mathbf{R}_{\alpha}-\mathbf{R}_{k}^{\beta}\right|} \\
& +\sum_{\alpha=A, B} \sum_{k} \sum_{\beta=\mathrm{Me}, \mathrm{Cl}} 4 \epsilon\left[\left(\frac{\sigma}{\left|\mathbf{R}_{\alpha}-\mathbf{R}_{k}^{\beta}\right|}\right)^{12}\right. \\
& \left.-\left(\frac{\sigma}{\left|\mathbf{R}_{\alpha}-\mathbf{R}_{k}^{\beta}\right|}\right)^{6}\right]
\end{aligned}
$$

with the LJ parameters $\sigma$ and $\epsilon$ chosen as $3.5 \AA$ and $200 \mathrm{~K}$, respectively. An important feature of the model is that the partial charges on the H-bonding complex, $q_{\alpha}(\alpha=A, H, B)$, changes greatly from the reactant covalent state $\left(q_{A}^{c}\right.$ $\left.=-0.5 e, q_{H}^{c}=+0.5 e, q_{B}^{c}=0\right)$ to the product ionic state $\left(q_{A}^{i}\right.$ $\left.=-1.0 e, q_{H}^{i}=+0.5 e, q_{B}^{i}=+0.5 e\right)$, which is represented by a smooth switching function as

$$
\begin{aligned}
& q_{\alpha}(r)=[1-f(r)] q_{\alpha}^{c}+f(r) q_{\alpha}^{i}, \\
& f(r)=\frac{1}{2}\left[1+\frac{r-r_{0}}{\sqrt{\left(r-r_{0}\right)^{2}+l^{2}}}\right]
\end{aligned}
$$

with $r_{0}=1.43 \AA$ and $l=0.125 \AA$. The product ionic state thus exhibits a large dipole moment and is stabilized strongly by the polar solvent. With the above potential functions the total Hamiltonian of the solution becomes
TABLE II. TIPS parameters for the methyl chloride solvent model.

\begin{tabular}{lccc}
\hline \hline Site & $q(e)$ & $A^{2}\left(\mathrm{kcal} \mathrm{mol}{ }^{-1} \AA^{12}\right)$ & $C^{2}\left(\mathrm{kcal} \mathrm{mol}^{-1} \AA^{6}\right)$ \\
\hline $\mathrm{CH}_{3}$ & +0.25 & $7.95 \times 10^{6}$ & 2750 \\
$\mathrm{Cl}$ & -0.25 & $5.25 \times 10^{6}$ & 2950 \\
\hline
\end{tabular}

$$
H=H_{\text {gas }}+T_{S}+V_{\mathrm{SS}}\left(\mathbf{R}_{S}\right)+V_{\mathrm{CS}}\left(r, \mathbf{R}_{A}, \mathbf{R}_{B}, \mathbf{R}_{S}\right),
$$

where $T_{S}$ is the translational and rotational kinetic energies of the solvent molecules.

\section{B. Quantum instanton theory for rate constants}

In this section we present the working expressions of the QI theory. ${ }^{18}$ The derivation of the QI rate expression begins with the following formally exact expression: ${ }^{16}$

$$
\begin{aligned}
k(T)= & \frac{1}{2 \pi \hbar Q_{A}} \int d E e^{-\beta E} \frac{(2 \pi \hbar)^{2}}{2} \\
& \times \operatorname{tr}[\hat{F} \delta(E-\hat{H}) \hat{F} \delta(E-\hat{H})],
\end{aligned}
$$

and uses a steepest descent approximation to establish an approximate relation between the microcanonical density operator $\delta(E-\hat{H})$ and the Boltzmann operator $e^{-\beta \hat{H} / 2}$. The integral over $E$ in Eq. (2.7) is also evaluated within the steepest descent approximation. $Q_{A}$ in Eq. (2.7) is the reactant partition function, $\beta$ is inverse temperature $1 /\left(k_{B} T\right)$, and $\hat{F}$ is a flux operator given by

$$
\hat{F}=\frac{1}{2 m_{H}}\left[\hat{p}_{r} \delta\left(\hat{r}-r^{\ddagger}\right)+\delta\left(\hat{r}-r^{\ddagger}\right) \hat{p}_{r}\right],
$$

where $p_{r}$ is the conjugate momentum of $r$. We note that the dividing surface is defined as $r=r^{\ddagger}$, i.e., using the proton coordinate $r$ rather than a collective solvent coordinate such as an energy gap function. This choice is simply to facilitate comparison with the result of Azzouz and Borgis. ${ }^{27,32} r^{\star}$ in Eq. (2.8) is an adjustable parameter that is chosen according to an appropriate stationary condition (see below). The approximations described above lead to the following QI expression: ${ }^{18}$

$$
k(T) \approx k_{\mathrm{QI}}=\frac{1}{Q_{A}} C_{\mathrm{ff}}(0) \frac{\sqrt{\pi} \hbar}{2} \frac{1}{\Delta H},
$$

where $C_{\mathrm{ff}}(0)$ is the zero time value of the flux-flux correlation function as defined by Miller et al., ${ }^{16}$

$$
C_{\mathrm{ff}}(t)=\operatorname{tr}\left[e^{-\beta \hat{H} / 2} \hat{F} e^{-\beta \hat{H} / 2} e^{i \hat{H} t / \hbar} \hat{F} e^{-i \hat{H} t / \hbar}\right],
$$

while $\Delta H$ is a particular type of energy variance given by

$$
\Delta H^{2}=\frac{\operatorname{tr}\left[\hat{H}^{2} e^{-\beta \hat{H} / 2} \delta\left(\hat{r}-r^{\ddagger}\right) e^{-\beta \hat{H} / 2} \delta\left(\hat{r}-r^{\ddagger}\right)\right]-\operatorname{tr}\left[\hat{H} e^{-\beta \hat{H} / 2} \delta\left(\hat{r}-r^{\ddagger}\right) \hat{H} e^{-\beta \hat{H} / 2} \delta\left(\hat{r}-r^{\ddagger}\right)\right]}{\operatorname{tr}\left[e^{-\beta \hat{H} / 2} \delta\left(\hat{r}-r^{\ddagger}\right) e^{-\beta \hat{H} / 2} \delta\left(\hat{r}-r^{\ddagger}\right)\right]},
$$


which can also be written as

$$
\Delta H=\hbar\left[-\frac{\ddot{C}_{\mathrm{dd}}(0)}{2 C_{\mathrm{dd}}(0)}\right]^{1 / 2}
$$

where $C_{\mathrm{dd}}(0)$ and $\ddot{C}_{\mathrm{dd}}(0)$ are the zero-time value and its second derivative, respectively, of the "delta-delta" correlation function,

$$
\begin{aligned}
C_{\mathrm{dd}}(t)= & \operatorname{tr}\left[e^{-\beta \hat{H} / 2} \delta\left(\hat{r}-r^{\ddagger}\right) e^{-\beta \hat{H} / 2} e^{i \hat{H} t / \hbar}\right. \\
& \left.\times \delta\left(\hat{r}-r^{\ddagger}\right) e^{-i \hat{H} t / \hbar}\right] .
\end{aligned}
$$

In practice the $\Delta H$ above is modified as follows to correct the free particle (or high-temperature) limit,

$$
\Delta H \rightarrow \Delta H+(\sqrt{\pi}-\sqrt{2}) / \beta,
$$

which is found to somewhat improve the QI rate. ${ }^{18}$

The location of the dividing surface, namely, the value of the parameter $r^{\ddagger}$ in Eq. (2.8), is determined by the following stationary condition: ${ }^{18,33}$

$$
\frac{\partial C_{\mathrm{dd}}(0)}{\partial r^{\ddagger}}=0 .
$$

This condition originates from a semiclassical consideration on the instanton trajectory, but it can also be regarded as an approximate condition for minimizing dynamical "recrossing" effects that cannot be captured by QTST. More precisely, according to Predescu and Miller, ${ }^{34} C_{\mathrm{ff}}(0)$ should be minimized with respect to $r^{\ddagger}$ in order to suppress an oscillatory behavior of $C_{\mathrm{ff}}(t)$ (or equivalently, to obtain the best accuracy of QTST), but $C_{\mathrm{dd}}(0)$ may be used instead because the latter exhibits essentially the same exponential behavior as a function of $r^{\ddagger}$. The use of $C_{\mathrm{dd}}(0)$ has the practical benefit that it is simpler to evaluate via standard statistical sampling methods (see below).

\section{Path integral evaluation of the quantum instanton rate}

The QI rate in Eq. (2.9) is expressed solely in terms of the Boltzmann operator and can be evaluated with imaginary time path integral techniques. ${ }^{9,10}$ In this section we present key steps of the implementation as well as the relevant path integral estimators. ${ }^{22}$ For simplicity of notation, hereafter we consider the classical (or single time-slice) limit of the bath coordinates, $\mathbf{R} \equiv\left(\mathbf{R}_{A}, \mathbf{R}_{B}, \mathbf{R}_{S}\right)$ (see $S e c$. II F for the quantum treatment of the latter).

To proceed, we rewrite the QI rate in Eq. (2.9) as

$$
k_{\mathrm{QI}}=p \nu,
$$

with

$$
p=\frac{C_{\mathrm{dd}}(0)}{Q_{A}}, \quad \nu=\frac{C_{\mathrm{ff}}(0)}{C_{\mathrm{dd}}(0)} \frac{\sqrt{\pi} \hbar}{2 \Delta H},
$$

where $p$ and $\nu$ are quantities similar in nature to the activation probability and the pre-exponential factor in the classical TST, respectively. The factor $p$ can be obtained with methods of rare events such as umbrella sampling or thermodynamic integration. To be specific, we discretize the Boltzmann operator in $C_{\mathrm{dd}}(0)$ and $Q_{A}$ using the standard procedure as

$$
p\left(r_{a}, r_{b}\right)=\frac{C_{\mathrm{dd}}\left(0 ; r_{a}, r_{b}\right)}{Q_{A}}=\frac{\int d \mathbf{R} \int d r^{(1)} \cdots \int d r^{(P)} \exp (-\beta \Phi) \delta\left(r^{(0)}-r_{a}\right) \delta\left(r^{(P / 2)}-r_{b}\right)}{\int d \mathbf{R} \int d r^{(1)} \cdots \int d r^{(P)} \exp (-\beta \Phi) h\left(r_{*}-r^{(0)}\right) h\left(r_{*}-r^{(P / 2)}\right)}
$$

where the proton coordinate $r$ is discretized into $P$ slices, $\left\{r^{(1)}, \ldots, r^{(P)}\right\}$, and the corresponding action is

$$
\Phi=\frac{m_{H} P}{2 \hbar^{2} \beta^{2}} \sum_{s=1}^{P}\left(r^{(s)}-r^{(s-1)}\right)^{2}+\frac{1}{P} \sum_{s=1}^{P} V\left(r^{(s)}, \mathbf{R}\right) .
$$

In Eq. (2.18), $h(x)$ is the Heaviside step function and $r_{*}$ is an adjustable parameter that roughly discriminates between the reactant and product regions. Note that $p\left(r_{a}, r_{b}\right)$ is defined such that the parameter $r^{\ddagger}$ can artificially take two independent values, $r_{a}$ and $r_{b}$, which leads to a simple normalization of $p\left(r_{a}, r_{b}\right)$ in the reactant space,

$$
\int_{-\infty}^{r_{*}} d r_{a} \int_{-\infty}^{r_{*}} d r_{b} p\left(r_{a}, r_{b}\right)=1 .
$$

The necessary value of $p$ is obtained by generating a normalized histogram of $\left(r^{(0)}, r^{(P / 2)}\right)$ and finding the stationary point of the "free energy" curve defined by

$$
W\left(r^{\prime}\right)=-\beta^{-1} \ln p\left(r^{\prime}\right)
$$

with $p\left(r^{\prime}\right)=p\left(r_{a}=r^{\prime}, r_{b}=r^{\prime}\right)$. The above procedure can be achieved, e.g., via two-dimensional umbrella sampling, where a bias (or umbrella) potential of $\left(r^{(0)}, r^{(P / 2)}\right)$ is added to the discretized action $\Phi$ in Eq. (2.19) and the bias is removed afterward from the resulting free energy. A particular version called adaptive umbrella sampling is used in Ref. 22 in the context of the QI theory. An alternative way of computing $p(r)$ is thermodynamic integration with respect to the reaction coordinate:

$$
p\left(r^{\ddagger}\right)=p\left(r_{A}\right) \exp \left[-\beta \int_{r_{A}}^{r^{\ddagger}} d r^{\prime} \frac{\partial W\left(r^{\prime}\right)}{\partial r^{\prime}}\right],
$$

where $r_{A}$ is an arbitrary constant that is chosen close to the minimum of the free energy curve in the reactant region, and the "mean force" $-\partial W\left(r^{\prime}\right) / \partial r^{\prime}$ is calculated via

$$
\frac{\partial W\left(r^{\prime}\right)}{\partial r^{\prime}}=\left\langle\frac{\partial \Phi}{\partial r^{(0)}}+\frac{\partial \Phi}{\partial r^{(P / 2)}}\right\rangle_{r^{\prime}},
$$

where the constrained path average is defined by 


$$
\langle\cdots\rangle_{r^{\prime}}=\frac{\int d \mathbf{R} \int d r^{(1)} \cdots \int d r^{(P)} \exp (-\beta \Phi) \delta\left(r^{(0)}-r^{\prime}\right) \delta\left(r^{(P / 2)}-r^{\prime}\right)(\cdots)}{\int d \mathbf{R} \int d r^{(1)} \cdots \int d r^{(P)} \exp (-\beta \Phi) \delta\left(r^{(0)}-r^{\prime}\right) \delta\left(r^{(P / 2)}-r^{\prime}\right)} .
$$

While the determination of the reference value $p\left(r_{A}\right)$ in Eq. (2.22) still requires a two-dimensional histogram calculation, no bias potential is necessary so long as $r_{A}$ is sufficiently close to the bottom of the reactant well. Due to this aspect the thermodynamic integration may be more straightforward than two-dimensional umbrella sampling in some situations (e.g., when a good bias potential is difficult to construct).

The factor $\nu$ in Eq. (2.16), on the other hand, is a quantity associated with the top of the activation barrier and can be obtained from appropriate constrained sampling. This factor consists of two ratios, $C_{\mathrm{ff}}(0) / C_{\mathrm{dd}}(0)$ and $\ddot{C}_{\mathrm{dd}}(0) / C_{\mathrm{dd}}(0)$ [via Eq. (2.12)]. The first one can be evaluated as

$$
\frac{C_{\mathrm{ff}}(0)}{C_{\mathrm{dd}}(0)}=\left\langle v^{(0)} v^{(P / 2)}\right\rangle_{\ddagger},
$$

where $\langle\cdots\rangle_{\ddagger}$ is the constrained path average in Eq. (2.24) with $r^{\prime}=r^{\ddagger}$, and the "velocity" factor $v^{(s)}$ is defined by

$$
v^{(s)}=\frac{i}{2 \hbar \Delta \beta}\left(r^{(s+1)}-r^{(s-1)}\right)
$$

with $\Delta \beta=\beta / P$. The statistical estimator in Eq. (2.25) is obtained by applying the flux operator in Eq. (2.8) directly onto the neighboring high-temperature Boltzmann operators, $\exp (-\Delta \beta \hat{H})$, in the discretized path integral expression for $C_{\text {ff }}(0)$. In contrast, the second ratio, $\ddot{C}_{\mathrm{dd}}(0) / C_{\mathrm{dd}}(0)$, can be obtained in several different ways. For example, one may express $\ddot{C}_{\mathrm{dd}}(0)$ as

$$
\begin{aligned}
\ddot{C}_{\mathrm{dd}}(0)= & -\operatorname{tr}\left\{e^{-\beta \hat{H} / 2} \frac{i}{\hbar}\left[\hat{H}, \delta\left(\hat{r}-r^{\ddagger}\right)\right] e^{-\beta \hat{H} / 2} \frac{i}{\hbar}\right. \\
& \left.\times\left[\hat{H}, \delta\left(\hat{r}-r^{\ddagger}\right)\right]\right\},
\end{aligned}
$$

and eliminate the kinetic energy operator in the commutator through integration by parts to give

$$
\frac{\ddot{C}_{\mathrm{dd}}(0)}{C_{\mathrm{dd}}(0)}=-\left\langle v^{(0)} v^{(P / 2)} w^{(0)} w^{(P / 2)}\right\rangle_{\ddagger},
$$

where $v^{(s)}$ is the velocity factor in Eq. (2.26), while $w^{(s)}$ is defined by

$$
w^{(s)}=\frac{m_{H}}{\hbar^{2} \Delta \beta}\left(2 r^{(s)}-r^{(s+1)}-r^{(s-1)}\right)+\Delta \beta \frac{\partial V\left(r^{(s)}, \mathbf{R}\right)}{\partial r^{(s)}} .
$$

An alternative method is to take the derivative of $C_{\mathrm{dd}}(t)$ along the imaginary-time axis (i.e., $t=-i \hbar \lambda$ ),

$$
\ddot{C}_{\mathrm{dd}}(0)=-\left.\frac{1}{\hbar^{2}} \frac{d^{2}}{d \lambda^{2}} C_{\mathrm{dd}}(-i \hbar \lambda)\right|_{\lambda=0},
$$

which gives

$$
\frac{\ddot{C}_{\mathrm{dd}}(0)}{C_{\mathrm{dd}}(0)}=-\frac{1}{\hbar^{2}}\left\langle F^{2}+G\right\rangle_{\ddagger}
$$

with

$$
F=\sum_{s=1}^{P}\left\{\frac{m_{H}}{2 \hbar^{2} \Delta \beta^{2}}\left(r^{(s)}-r^{(s-1)}\right)^{2}-\bar{V}^{(s)}\right\} c^{(s)}
$$

and

$$
G=\sum_{s=1}^{P}\left\{\frac{1}{2 \Delta \beta^{2}}-\frac{m_{H}}{\hbar^{2} \Delta \beta^{3}}\left(r^{(s)}-r^{(s-1)}\right)^{2}\right\}\left(c^{(s)}\right)^{2},
$$

where $\bar{V}^{(s)}=\left[V\left(r^{(s)}, \mathbf{R}\right)+V\left(r^{(s-1)}, \mathbf{R}\right)\right] / 2$, and the coefficients $c^{(s)}$ are defined by

$$
c^{(s)}=\left\{\begin{array}{l}
1 /(P / 2-2), \quad 1<s<P / 2 \\
-1 /(P / 2-2), \quad P / 2+1<s<P, \\
0, \quad \text { otherwise }
\end{array}\right.
$$

whose derivation is summarized in Appendix B. We refer to the above two estimators for $\ddot{C}_{\mathrm{dd}}(0) / C_{\mathrm{dd}}(0)$ in Eqs. (2.28) and (2.31) as the "direct" and "thermodynamic" estimators, respectively, following the conventions in the literature ${ }^{10}$ [note that $\lambda$ in Eq. (2.30) has the same physical units as $\beta$ $\left.=1 / k_{B} T\right]$. As is often the case, the two estimators exhibit quite different statistical behavior, which will be examined numerically in Sec. III C.

\section{Other path integral quantum transition state theory}

As mentioned in the Introduction, several different types of QTST already exist, and it is thus informative to compare the results of the QI theory with some of the other versions. To this end we consider the following two QTSTs that are directly amenable to path integral evaluation. The first is the centroid-density QTST that defines the activation free energy in terms of the centroid of the imaginary-time path, ${ }^{11-14}$

$$
k(T) \simeq k_{c}(T)=\frac{1}{\sqrt{2 \pi m_{H} \beta}} p_{c}\left(r_{c}^{\ddagger}\right),
$$

where the centroid density $p_{c}$ is given by

$$
p_{c}\left(r_{c}\right)=\frac{\int d \mathbf{R} \int d r^{(1)} \cdots \int d r^{(P)} \exp (-\beta \Phi) \delta\left(\tilde{r}-r_{c}\right)}{\int d \mathbf{R} \int d r^{(1)} \cdots \int d r^{(P)} \exp (-\beta \Phi) h\left(r_{*}-\widetilde{r}\right)}
$$

with the path centroid $\widetilde{r}$ being

$$
1 / P \sum_{s=1}^{P} r^{(s)} \text {. }
$$

The value of $r_{c}^{*}$ in Eq. (2.34) is chosen such that it minimizes the centroid density $p_{c}\left(r_{c}\right)$. The above method can be imple- 
mented rather straightforwardly and has already been applied to numerous condensed-phase reactions including the Azzouz-Borgis model in Sec. II A.

The second QTST of interest is that proposed by Hansen and Andersen, ${ }^{15}$ which employs the following short-time approximation to the flux-flux correlation function in Eq. (2.10),

$$
C_{\mathrm{ff}}(t) \approx C_{\mathrm{ff}}^{\mathrm{HA}}(t)=\frac{a e^{b t^{2}}}{\left[t^{2}+(\hbar \beta / 2)^{2}\right]^{3 / 2}}
$$

with

$$
a=\left(\frac{\hbar \beta}{2}\right)^{3} C_{\mathrm{ff}}(0), \quad b=\frac{6}{(\hbar \beta)^{2}}+\frac{\ddot{C}_{\mathrm{ff}}(0)}{2 C_{\mathrm{ff}}(0)} .
$$

The corresponding approximate rate is given by

$$
k(T) \approx k_{\mathrm{HA}}(T)=\frac{1}{Q_{A}} \int_{0}^{\infty} d t C_{\mathrm{ff}}^{\mathrm{HA}}(t) .
$$

This method is based on a Padé approximation to $d \ln \left[C_{\mathrm{ff}}\left(z^{1 / 2}\right)\right] / d z$, and it reproduces the correct short-time behavior of the true $C_{\mathrm{ff}}(t)$, satisfies known analyticity properties of the latter, and becomes exact for a free particle. Despite these appealing features the above method has not been utilized in realistic condensed-phase problems because of the lack of practical implementation schemes. Here we find that such a scheme can be established in a straightforward manner by rewriting Eq. (2.38) as

$$
k_{\mathrm{HA}}(T)=\frac{C_{\mathrm{dd}}(0)}{Q_{A}}\left\{\frac{C_{\mathrm{ff}}(0)}{C_{\mathrm{dd}}(0)} \int_{0}^{\infty} d t \frac{(\hbar \beta / 2)^{3} e^{b t^{2}}}{\left[t^{2}+(\hbar \beta / 2)^{2}\right]^{3 / 2}}\right\},
$$

and evaluating the ratios $C_{\mathrm{dd}}(0) / Q_{A}, C_{\mathrm{ff}}(0) / C_{\mathrm{dd}}(0)$, and $\ddot{C}_{\mathrm{ff}}(0) / C_{\mathrm{ff}}(0)$ [in coefficient $b$ in Eq. (2.37)] using the present path integral approach in Sec. IIC. An ingredient required here is the statistical estimator for $\ddot{C}_{\mathrm{ff}}(0) / C_{\mathrm{ff}}(0)$, which can be generated by combining the estimators for $C_{\mathrm{ff}}(0) / C_{\mathrm{dd}}(0)$ in Eq. $(2.25)$ and that for $\ddot{C}_{\mathrm{dd}}(0) / C_{\mathrm{dd}}(0)$ in Eq. (2.31) as follows (see Appendix B for more details):

$$
\frac{\ddot{C}_{\mathrm{ff}}(0)}{C_{\mathrm{ff}}(0)}=-\frac{1}{\hbar^{2}} \frac{\left\langle v^{(0)} v^{(P / 2)}\left(F^{2}+G\right)\right\rangle_{\ddagger}}{\left\langle v^{(0)} v^{(P / 2)}\right\rangle_{\ddagger}} .
$$

Thus the Hansen-Andersen QTST can be evaluated simultaneously with the present QI theory.

\section{E. Direct evaluation of the kinetic isotope effect}

The $H / D$ KIE, $k\left(m_{H}\right) / k\left(m_{D}\right)$, provides an experimentally important clue to understand the degree of nuclear quantum effects in a given reaction. A conventional way of estimating the KIE by computer simulation is to calculate the absolute rates $k\left(m_{H}\right)$ and $k\left(m_{D}\right)$ separately, but one can also evaluate the ratio $k\left(m_{H}\right) / k\left(m_{D}\right)$ directly without generating the absolute rates. In this section we describe such an approach based on thermodynamic integration of the rate with respect to hydrogen mass. The primary benefit of this method is that one can avoid costly evaluation of the activation free energy for the $H$ and $D$ transfer.
The KIE is thus given in terms of the QI rate of Eq. (2.16) as follows:

$$
\frac{k_{\mathrm{QI}}\left(m_{H}\right)}{k_{\mathrm{QI}}\left(m_{D}\right)}=\frac{p\left(m_{H}\right) \nu\left(m_{H}\right)}{p\left(m_{D}\right) \nu\left(m_{D}\right)},
$$

where the dependence of relevant quantities on hydrogen mass $m$ is indicated. Our strategy is to calculate the ratio $p\left(m_{H}\right) / p\left(m_{D}\right)$ directly via thermodynamic integration with respect to $m$. [We note that the evaluation of $p$ is more costly than $\nu$ because the former requires umbrella sampling etc., while the latter can be obtained from a single constrained average in Eq. (2.24) with $r^{\prime}=r^{\ddagger}$.] As such, we rewrite the ratio of $p$ as

$$
\frac{p\left(m_{H}\right)}{p\left(m_{D}\right)}=\exp \left\{-\int_{m_{H}}^{m_{D}} d m \frac{\partial}{\partial m} \ln p(m)\right\},
$$

and by substituting the discretized path integral expression for $p(m)$ in Eq. (2.18) into the log derivative of $p(m)$, we have

$$
\frac{\partial}{\partial m} \ln p(m)=\frac{\beta}{m}\left[\langle K(m)\rangle_{\ddagger}-\langle K(m)\rangle_{A}\right],
$$

where $K(m)$ is the thermodynamic estimator for the kinetic energy, ${ }^{10}$

$$
K(m)=\frac{P}{2 \beta}-\frac{m P}{2 \hbar^{2} \beta^{2}} \sum_{s=1}^{P}\left(r^{(s)}-r^{(s-1)}\right)^{2} .
$$

The bracket $\langle\cdots\rangle_{\ddagger}$ in Eq. (2.43) has the same meaning as in Eq. (2.25), while $\langle\cdots\rangle_{A}$ is the equilibrium path average in the reactant space defined by

$$
\langle\cdots\rangle_{A}=\frac{\int d \mathbf{R} \int d r^{(1)} \cdots \int d r^{(P)} \exp (-\beta \Phi) h_{A}(\cdots)}{\int d \mathbf{R} \int d r^{(1)} \cdots \int d r^{(P)} \exp (-\beta \Phi) h_{A}}
$$

with $h_{A}=h\left(r_{*}-r^{(0)}\right) h\left(r_{*}-r^{(P / 2)}\right)$. Thus, the KIE is expressed in terms of the average kinetic energy in the reactant and barrier regions as follows:

$$
\begin{aligned}
\frac{k_{\mathrm{QI}}\left(m_{H}\right)}{k_{\mathrm{QI}}\left(m_{D}\right)}= & \frac{\nu\left(m_{H}\right)}{\nu\left(m_{D}\right)} \\
& \times \exp \left\{\beta \int_{m_{H}}^{m_{D}} d m\left[\langle K(m)\rangle_{A}-\langle K(m)\rangle_{\sharp}\right] / m\right\} .
\end{aligned}
$$

An important point here is that $\langle K(m)\rangle$ is generally a slowly varying function of $m$, suggesting that a simple quadrature with only a few points should suffice for practical purposes. This feature will be illustrated numerically in Sec. III D. We note also that the above procedure is equally applicable to a wide class of other rate theories including the centroiddensity and Hansen-Andersen QTST.

\section{F. Quantizing the bath degrees of freedom}

All the path integral expressions in the preceding sections were presented in the classical (i.e., single time-slice) limit of the bath degrees of freedom, $\mathbf{R}=\left(\mathbf{R}_{A}, \mathbf{R}_{B}, \mathbf{R}_{S}\right)$. While this approximation seems excellent due to the large masses associated with $\mathbf{R}$, one can examine its accuracy with 
minimal computational effort by using only a few time slices for $\mathbf{R}$, following the procedure described in Ref. 35: Specifically, the original "beads" for $\mathbf{R}$, namely $\left\{\mathbf{R}^{(1)}, \ldots, \mathbf{R}^{(P)}\right\}$, are first grouped into $P^{\prime}(<P)$ segments of length $L$, where the $\sigma$ th segment consists of $\mathbf{R}^{(s)}$ with $s_{0}+L \sigma \leqslant s<s_{0}+L(\sigma$ +1 ), and $s_{0}$ is an arbitrary integer offset. For convenience, the first bead in each segment is denoted as $\mathbf{R}^{[\sigma]}$ $\equiv \mathbf{R}^{\left(s_{0}+L \sigma\right)}$. Since the bath degrees of freedom in the present system are close to classical entities, the separation between neighboring beads, $\mathbf{R}^{(s)}$ and $\mathbf{R}^{(s-1)}$, remains extremely small, and thus one may neglect the dependence of the potential function on $\left\{\mathbf{R}^{(s)}\right\}$ within a given segment,

$$
V\left(r^{(s)}, \mathbf{R}^{(s)}\right) \approx V\left(r^{(s)}, \mathbf{R}^{[\sigma(s)]}\right)
$$

with $\sigma(s)=\operatorname{int}\left[\left(s-s_{0}\right) / L\right]$. This approximation permits all $\mathbf{R}^{(s)}$ except for $\mathbf{R}^{[\sigma(s)]}$ to be integrated out from the original path integral, which leads to a modified expression for the partition function of the form

$$
\begin{aligned}
Q= & \int d r^{(1)} \cdots \int d r^{(P)} \int d \mathbf{R}^{[1]} \ldots \int d \mathbf{R}^{\left[P^{\prime}\right]} \\
& \times\left[\prod_{s=1}^{P}\left\langle r^{(s)}\left|e^{-\beta \hat{T}_{r} / P}\right| r^{(s-1)}\right\rangle\right] \\
& \times\left[\prod_{\sigma=1}^{P^{\prime}}\left\langle\mathbf{R}^{[\sigma]}\left|e^{-\beta \hat{T}_{R} / P^{\prime}}\right| \mathbf{R}^{[\sigma-1]}\right\rangle\right] \\
& \times \exp \left\{-\frac{\beta}{P} \sum_{s=1}^{P} V\left(r^{(s)}, \mathbf{R}^{[\sigma(s)]}\right)\right\}
\end{aligned}
$$

where $\hat{T}_{r}$ and $\hat{T}_{R}$ is the kinetic energy operators for $r$ and $\mathbf{R}$, respectively.

Other path integral expressions in Sec. II C can also be generalized through similar arguments. One exception that requires care is the thermodynamic estimator for $\ddot{C}_{\mathrm{dd}}(0) / C_{\mathrm{dd}}(0)$ in Eq. (2.31), where the $F$ and $G$ functions in Eq. (2.32) are modified as follows:

$$
\begin{aligned}
F= & \sum_{s=1}^{P}\left\{\frac{m_{H}}{2 \hbar^{2} \Delta \beta^{2}}\left(r^{(s)}-r^{(s-1)}\right)^{2}-\bar{V}^{(s)}\right\} c^{(s)} \\
& +\sum_{\sigma=1}^{P^{\prime}}\left\{\frac{M_{A}}{2 \hbar^{2}\left(\Delta \beta^{\prime}\right)^{2}}\left(\mathbf{R}_{A}^{[\sigma]}-\mathbf{R}_{A}^{[\sigma-1]}\right)^{2}\right. \\
& \left.+\frac{M_{B}}{2 \hbar^{2}\left(\Delta \beta^{\prime}\right)^{2}}\left(\mathbf{R}_{B}^{[\sigma]}-\mathbf{R}_{B}^{[\sigma-1]}\right)^{2}\right\} C^{[\sigma]}
\end{aligned}
$$

and

$$
\begin{aligned}
G= & \sum_{s=1}^{P}\left\{\frac{f_{r}}{2(\Delta \beta)^{2}}-\frac{m_{H}}{\hbar^{2}(\Delta \beta)^{3}}\left(r^{(s)}-r^{(s-1)}\right)^{2}\right\}\left(c^{(s)}\right)^{2} \\
& +\sum_{\sigma=1}^{P^{\prime}}\left\{\frac{f_{A B}}{2\left(\Delta \beta^{\prime}\right)^{2}}-\frac{M_{A}}{\hbar^{2}\left(\Delta \beta^{\prime}\right)^{3}}\left(\mathbf{R}_{A}^{[\sigma]}-\mathbf{R}_{A}^{[\sigma-1]}\right)^{2}\right. \\
& \left.-\frac{M_{B}}{\hbar^{2}\left(\Delta \beta^{\prime}\right)^{3}}\left(\mathbf{R}_{B}^{[\sigma]}-\mathbf{R}_{B}^{[\sigma-1]}\right)^{2}\right\}\left(C^{[\sigma]}\right)^{2}
\end{aligned}
$$

with $\Delta \beta=\beta / P$ and $\Delta \beta^{\prime}=\beta / P^{\prime}$. The numbers of partial degrees of freedom $f_{r}$ and $f_{A B}$ in Eq. (2.49b) are 1 and 6, respectively, while the average potentials $\bar{V}^{(s)}$ and the contracted coefficients $C^{[\sigma]}$ are defined by

$$
\begin{aligned}
\bar{V}^{(s)}= & \frac{1}{2}\left\{V\left(r^{(s)}, \mathbf{R}_{A}^{[\sigma(s)]}, \mathbf{R}_{B}^{[\sigma(s)]}, \mathbf{R}_{S}\right)\right. \\
& \left.+V\left(r^{(s-1)}, \mathbf{R}_{A}^{[\sigma(s-1)]}, \mathbf{R}_{B}^{[\sigma(s-1)]}, \mathbf{R}_{S}\right)\right\}
\end{aligned}
$$

and

$$
C^{[\sigma]}=\sum_{s=s_{0}+L(\sigma-1)+1}^{s_{0}+L \sigma} c^{(s)}
$$

(see Appendix B for the derivation). Here we should emphasize that the quantum solvent contributions are neglected in Eq. (2.49). Although it is formally possible to include the solvent terms, it is not attempted in this paper because the resulting estimator may exhibit prohibitively large statistical errors. In the subsequent calculations, therefore, we use the above $F$ and $G$ functions approximately when the solvent is quantized as well as the proton and the donor-acceptor modes. [Note, however, that all the other quantities such as $p$ in Eq. (2.16) are treated rigorously, including the quantization of the solvent.]

\section{RESULTS AND DISCUSSIONS}

\section{A. Computational details}

The simulation conditions in this paper were chosen identical to those in Ref. 28. Specifically, the simulation was performed with periodic boundary condition for a cubic box of length $28 \AA$ at a temperature of $249 \mathrm{~K}$, where the box contained one reactive AHB complex and 255 solvent molecules with the density being $\rho=0.012 \AA^{-3}$. To facilitate comparison with the previous studies, the Coulombic potential for the solvent-solvent and solvent-complex interactions was smoothly truncated at $13.8 \AA$ using the Steinhauser function. $^{28}$

In the path integral calculations, the Boltzmann operator was discretized using the primitive approximation, $e^{-\epsilon H}$ $\approx e^{-\epsilon V / 2} e^{-\epsilon T} e^{-\epsilon V / 2}$, for all the degrees of freedom. Since the solvent in the Azzouz-Borgis model is a collection of rigid rotors, the following rotational density matrix was used in conjunction with the primitive approximation: ${ }^{36}$

$$
\begin{aligned}
\left\langle\mathbf{\Omega}^{\prime \prime}\left|\exp \left(-\epsilon \hat{T}_{\text {rot }}\right)\right| \mathbf{\Omega}^{\prime}\right\rangle= & \sum_{l=0}^{\infty}\left(\frac{2 l+1}{4 \pi}\right) \\
& \times \exp \left[-\epsilon \frac{l(l+1) \hbar^{2}}{2 I}\right] P_{l}\left(\boldsymbol{\Omega}^{\prime \prime} \cdot \boldsymbol{\Omega}^{\prime}\right),
\end{aligned}
$$

where $T_{\text {rot }}$ is the rotational kinetic energy of a rigid rotor, $\boldsymbol{\Omega}$ and $I$ is its orientational unit vector and inertia moment, respectively, and $P_{l}(x)$ is the Legendre polynomial. Since the above matrix element depends only on $\cos \gamma \equiv \boldsymbol{\Omega}^{\prime \prime} \cdot \boldsymbol{\Omega}^{\prime}$, we prepared a numerical table of the matrix element and interpolated it as necessary during the simulation.

The sampling of discretized paths was performed with Monte Carlo methods. The proton path was sampled effi- 


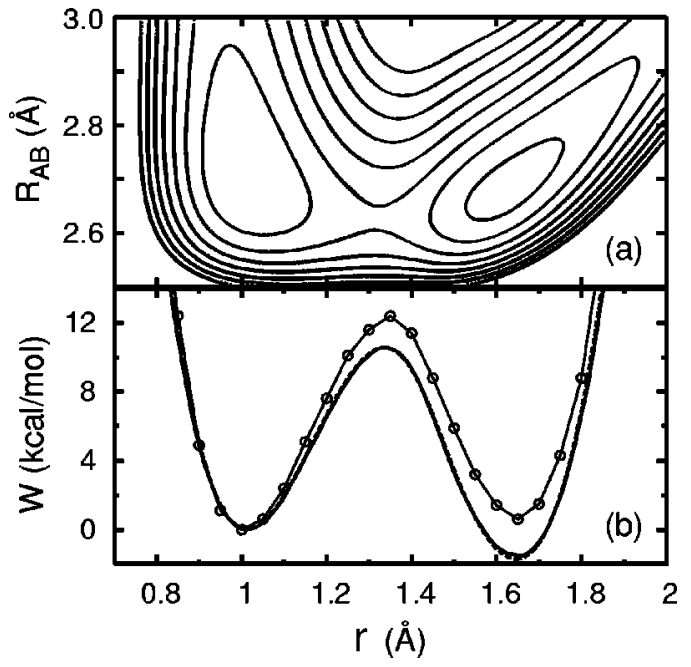

FIG. 2. Classical free energy surface associated with the probability density $p_{c l}\left(r, R_{A B}\right)$ in Eq. (3.2). Zero of energy is taken as the minimum of the reactant well. (a) Contours are plotted for energies of $0,4, \ldots, 28 \mathrm{kcal} / \mathrm{mol}$; (b) section at $R_{A B}=2.7 \AA$. The present result is shown by solid line, while the result obtained by McRae et al. (Ref. 31) using two-dimensional thermodynamic integration is shown by dashed line (almost indistinguishable from the present result). The corresponding free energy curve of Azzouz and Borgis is reproduced from Fig. 4 in Ref. 27 (line with circles).

ciently with the staging algorithm. ${ }^{37-39}$ For the rotational motion of the solvent, no such algorithms exist that can sample the rotational kinetic action exactly. We thus utilized the multiple "time step" Monte Carlo method of Hetényi et al.,$^{40}$ where the total potential is separated into a rapidly varying part $V_{\mathrm{I}}$ and a slowly varying part $V_{\mathrm{II}}$. This method performs several successive moves for $V_{I}$ and accepts or rejects the resulting configuration according to $V_{\mathrm{II}}$ in order to make the evaluation of $V_{\text {II }}$ less frequent than otherwise. In the present paper we chose the rapidly varying part as the rotational density matrix in Eq. (3.1) and the slowly varying part as the potential energy terms in the path integral action. This choice resulted in substantial savings in CPU time because the evaluation of the total potential is much more costly than that of the rotational density matrix.

The numbers of time slices, i.e., $P$ for the proton and $P^{\prime}$ for the bath degrees of freedom, were set to $\left(P, P^{\prime}\right)$ $=(40,4)$ in all the path integral simulations. This combination was found to be sufficient for reducing the discretization errors in the relevant quantities to $<5 \%$. The number of Monte Carlo cycles was set to $2-4 \times 10^{6}$ for computing a single ensemble average, which converged most of the ratios such as $C_{\mathrm{ff}}(0) / C_{\mathrm{dd}}(0)$ within $<10 \%$ statistical errors.

\section{B. Classical and quantum free energy surfaces}

Figure 2 displays the classical free energy surface associated with the probability density

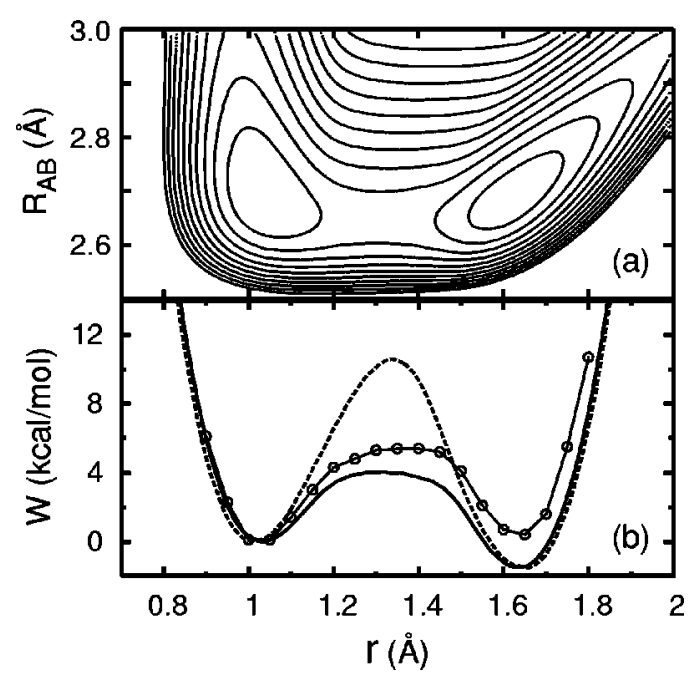

FIG. 3. Centroid free energy surface associated with the probability density $p_{c}\left(r, R_{A B}\right)$ in Eq. (3.3). Zero of energy is taken as the minimum of the reactant well. (a) Contours are plotted for energies of $0,2, \ldots, 20 \mathrm{kcal} / \mathrm{mol}$; (b) section at $R_{A B}=2.7 \AA$. The present result is shown by solid line. The corresponding free energy curve of Azzouz and Borgis is reproduced from Fig. 9(a) in Ref. 27 (line with circles). The classical free energy in Fig. 2 is also plotted for comparison [dashed line in panel (b)].

$$
p_{c l}\left(r^{\prime}, R_{A B}^{\prime}\right)=\frac{\int d \mathbf{R} \int d r e^{-\beta V} \delta\left(r-r^{\prime}\right) \delta\left(R_{A B}-R_{A B}^{\prime}\right)}{\int d \mathbf{R} \int d r e^{-\beta V}},
$$

where $V$ is the total potential of the system. The above density was generated using a simplified version of the adaptive umbrella sampling methods, ${ }^{22}$ where 20 sets of short simulations with $10^{5}$ Monte Carlo cycles were performed iteratively to construct a good global bias potential, and a long simulation with $6 \times 10^{6}$ cycles was run with the resulting bias potential to obtain Fig. 2. The number of bins for $r$ and $R_{A B}$ were set to 100 and 50, respectively. Other free energy surfaces in this section were also generated with the same procedure. The classical free energy in Fig. 2 exhibits a deep product well that is absent from the gas-phase potential in Fig. 1, which results from electrostatic stabilization of the ionic product by the polar solvent. Figure 2 compares the present classical free energy (solid line) with that obtained by McRae et al. ${ }^{31}$ (dashed line) and Azzouz and Borgis ${ }^{27}$ (circles), from which it is seen that the free energy of McRae et al. agrees almost perfectly with the present result while that of Azzouz and Borgis has a barrier height $\sim 2 \mathrm{kcal} / \mathrm{mol}$ higher than the present one. This discrepancy is probably due to the slightly different parametrization of the gas-phase potential $V_{H B}$ in Refs. 27 and 28, as manifested in Fig. 1. The difference in the gas-phase potential also affects the centroid free energy surface displayed in Fig. 3, where the associated probability density is defined as

$$
p_{c}\left(r_{c}, R_{A B}^{\prime}\right)=\frac{\int d \mathbf{R} \int d r^{(1)} \cdots \int d r^{(P)} \exp (-\beta \Phi) \delta\left(\tilde{r}-r_{c}\right) \delta\left(R_{A B}-R_{A B}^{\prime}\right)}{\int d \mathbf{R} \int d r^{(1)} \cdots \int d r^{(P)} \exp (-\beta \Phi)}
$$




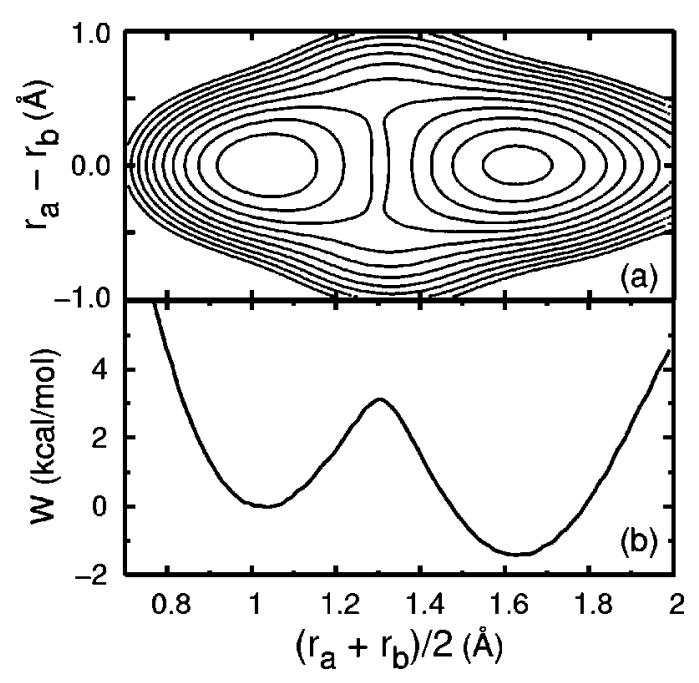

FIG. 4. Quantum free energy surface associated with the probability density $p\left(r_{a}, r_{b}\right)$ in Eq. (2.18). Zero of energy is taken as the minimum of the reactant well. (a) Contours are plotted for energies of -2 , $-1, \ldots, 10 \mathrm{kcal} / \mathrm{mol}$; (b) section at $r_{a}=r_{b}$ that corresponds to a single dividing surface.

Although there are other factors that may contribute to the difference in the free energy surfaces (e.g., details of the path integral calculation), we think that the most dominant factor would be the gas-phase potential as shown above.

Next, we plot in Fig. 4 the quantum free energy surface relevant to the QI theory, i.e., which defined in terms of the probability density $p\left(r_{a}, r_{b}\right)$ in Eq. (2.18). This free energy surface exhibits a double-well structure in the direction of $\left(r_{a}+r_{b}\right) / 2$, as in the classical and centroid cases, while it grows nearly quadratically with increasing $\left|r_{a}-r_{b}\right|$ (see Ref. 22 for the interpretation of this behavior). The QI rate is calculated using the dividing surface corresponding to the top of the free energy curve in Fig. 4(b), i.e., with $r_{a}=r_{b}$ $=r^{\ddagger}=1.30 \AA$ in this particular example. We note in addition that although the latter potential curve was obtained from two-dimensional umbrella sampling, it can equally be generated via thermodynamic integration of the factor $p$ with respect to the reaction coordinate, as described in Sec. II C.

\section{Statistical convergence of path integral estimators}

The QI theory requires the evaluation of several ratios such as $C_{\mathrm{ff}}(0) / C_{\mathrm{dd}}(0)$, which can be obtained simultaneously from a constrained path average in Eq. (2.24) with $r^{\prime}=r^{\ddagger}$. A practically important issue here is the rate of statistical convergence of these estimators, because it directly affects the computational time required. Figure 5 illustrates the convergence behavior of relevant estimators, where each panel contains results of three independent runs. From this figure it is seen that the estimator for $C_{\mathrm{ff}}(0) / C_{\mathrm{dd}}(0)$ in Eq. (2.25) [panel (a)] converges very rapidly and the ratio can be obtained quite accurately with minimal numerical effort. Next, comparing two types of estimators for $\ddot{C}_{\mathrm{dd}}(0) / C_{\mathrm{dd}}(0)$ [panel (b)], we see that the "thermodynamic" estimator in Eq. (2.31) (solid line) converges as rapidly as the estimator for $C_{\mathrm{ff}}(0) / C_{\mathrm{dd}}(0)$ in panel (a), whereas the direct estimator in Eq. (2.28) (dashed line) exhibits drastically large statistical
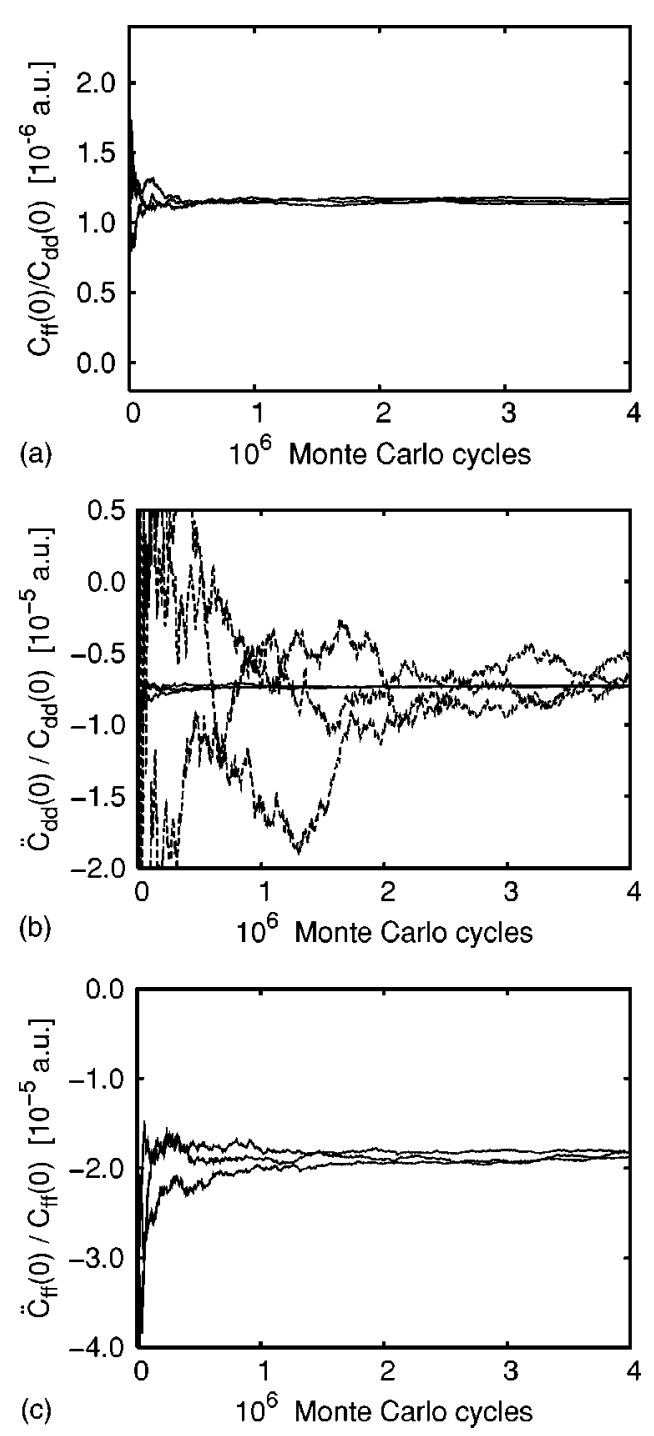

FIG. 5. Statistical convergence of path integral estimators for the QI theory and the Hansen-Andersen QTST. Results of three independent runs are plotted in each panel as a function of Monte Carlo cycles. (a) The estimator for $C_{\mathrm{ff}}(0) / C_{\mathrm{dd}}(0)$ in Eq. (2.25); (b) the "thermodynamic" estimator for $\ddot{C}_{\mathrm{dd}}(0) / C_{\mathrm{dd}}(0)$ in Eq. (2.31) (solid line) and the direct estimator for $\ddot{C}_{\mathrm{dd}}(0) / C_{\mathrm{dd}}(0)$ in Eq. (2.28) (dashed line); (c) the estimator for $\ddot{C}_{\mathrm{ff}}(0) / C_{\mathrm{ff}}(0)$ in Eq. $(2 \cdot 40)$.

errors. This difference in convergence rate stems partially from different information content in the two estimators, i.e., the direct estimator is based exclusively on the time slices near $s=0$ and $P / 2$ through the $v^{(s)}$ and $w^{(s)}$ factors, while the thermodynamic estimator utilizes all the time slices in an equivalent manner. We also find that the direct estimator is not favorable for an extrapolation procedure to the limit $P$ $\rightarrow \infty$ because its statistical error grows too rapidly with increase in $P$ (not illustrated in Fig. 5). Finally, the estimator for $\ddot{C}_{\mathrm{fff}}(0) / C_{\mathrm{ff}}(0)$ in Eq. (2.40) [panel (c)] converges somewhat more slowly than the thermodynamic estimator for $\ddot{C}_{\mathrm{dd}}(0) / C_{\mathrm{dd}}(0)$ because of the additional velocity factors. Thus, the Hansen-Andersen QTST is computationally slightly more costly than the QI theory if the same statistical accuracy is to be obtained. 
TABLE III. Rate constants (in unit of $10^{10} \mathrm{~s}^{-1}$ ) for the $\mathrm{H}$ and D transfer and H/D KIEs calculated with the quantum instanton theory, the HansenAndersen QTST, and the centroid-density QTST. Results of three quantization schemes (I)-(III) are compared, where scheme (I) quantizes the proton only, scheme (II) quantizes the proton and the donor-acceptor modes, and scheme (III) quantizes the whole solution.

\begin{tabular}{lccc}
\hline \hline \multicolumn{1}{c}{ Method } & H & D & H/D KIE \\
\hline & Scheme (I) & & \\
Quantum instanton & 21 & 0.48 & 44 \\
Hansen-Andersen & 16 & 0.45 & 36 \\
Centroid density & 14 & 0.38 & 37 \\
& Scheme (II) & & \\
Quantum instanton & 17 & 0.37 & 46 \\
Hansen-Andersen & 13 & 0.34 & 38 \\
Centroid density & 11 & 0.27 & 41 \\
& & & \\
Quantum instanton & Scheme (III) & & \\
Hansen-Andersen & 17 & 0.36 & 47 \\
Centroid density & 13 & 0.33 & 39 \\
\hline \hline
\end{tabular}

\section{Rate constants and $H / D$ kinetic isotope effects}

Table III summarizes the rate constants obtained with the QI theory as well as the centroid-density and HansenAndersen QTST. ${ }^{41}$ This table contains results of the three quantization schemes (I)-(III), where scheme (I) quantizes the proton only, scheme (II) includes the donor-acceptor modes $\left(\mathbf{R}_{A}, \mathbf{R}_{B}\right)$ into the quantum treatment, and scheme (III) quantizes the whole solution. Note that scheme (I) corresponds to the treatment by Azzouz and $\mathrm{Borgis}^{27}$ and Hammes-Schiffer and Tully, ${ }^{28}$ while scheme (II) broadly to McRae et al. ${ }^{31}$ and Kim and Hammes-Schiffer. ${ }^{29}$ Comparing the rates within a given quantization scheme, we find that the three QTSTs provide very similar estimates of the rate, within $\sim 30 \%$ of each other, which is not trivial if one recalls the totally different derivations of the three QTSTs. Next, comparison of different quantization schemes reveals that the quantization of donor-acceptor modes reduces the rate by $20 \%-30 \%$, which is in agreement with the results obtained by McRae et al. and primarily due to the zero-point energy difference between the reactant and transition state regions. Table III also shows that the quantum effect of the solvent is even smaller, which is to be expected because the present solvent model involves no "fast" degrees of freedom such as methane hydrogens or electronic polarizability. ${ }^{4}$ The small quantum effect of the bath modes hence validates the use of mixed quantum/classical approximations in the previous studies.

The H/D KIE in Table III was calculated with the absolute rates for the $H$ and $D$ transfer. As discussed in Sec. IIE, it can also be obtained through thermodynamic integration with respect to hydrogen mass. Figure 6 displays the key quantities in Eq. (2.46), namely, the average kinetic energy in the reactant and barrier regions, $\langle K(m)\rangle_{A}$ and $\langle K(m)\rangle_{\ddagger}$, as a function of hydrogen mass $m$. From this figure it is seen that the mass dependence of $\langle K(m)\rangle$ is very weak and close to linear in $m$. Thus only a few points of $m$ should suffice for accurate discretization of the mass integral in Eq. (2.46), leading to considerable savings in CPU time if one is inter-

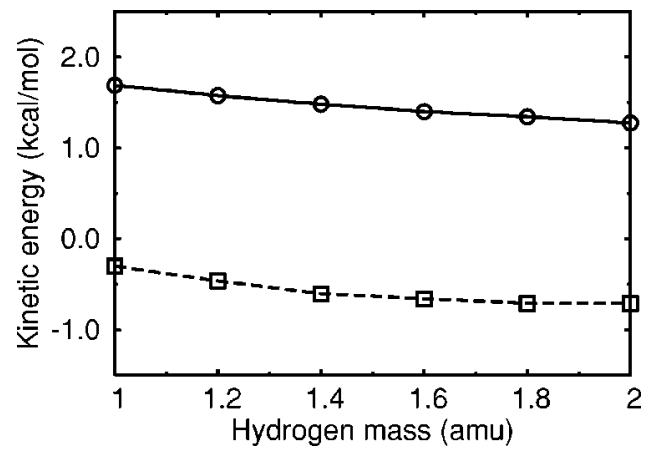

FIG. 6. Average kinetic energies $\langle K(m)\rangle_{A}$ (solid line with circles) and $\langle K(m)\rangle_{\ddagger}$ (dashed line with squares) as a function of hydrogen mass $m$, which is related to the KIE via Eq. (2.46).

ested only in the KIE itself rather than the absolute rates, since no calculation of activation free energy is required.

Table IV compares the present QTST rates with those obtained previously by other groups. ${ }^{42}$ Rows two and three contain the rates calculated by Azzouz and Borgis using a semiclassical curve-crossing TST and the centroid-density QTST. A critical point here is that their centroid rates (row three) are more than one order of magnitude smaller than our result (the last row) despite the fact that the same methodology has been used. As discussed above, this discrepancy presumably originates from the gas-phase potential $V_{H B}$ in Eq. (2.2). Because all the other studies listed in Table IV (including the present work) were performed with the parametrization of $V_{H B}$ by Hammes-Schiffer and Tully, it follows that the absolute rates in rows two and three should not be compared directly with the remaining rates. Now focusing on the other rows in the table, we find that the present QTST results agree reasonably well with the results of MDQT and VTST/MT within a factor of $\sim 2(7)$ for the $H(D)$ transfer.

TABLE IV. Comparison of the present QTST rates with the results of previous studies.

\begin{tabular}{|c|c|c|c|}
\hline Method & $\mathrm{H}$ & $\mathrm{D}$ & H/D KIE \\
\hline Classical TST ${ }^{\mathrm{a}}$ & $7.5 \times 10^{-4}$ & $5.3 \times 10^{-4}$ & 1.4 \\
\hline Curve-crossing $\mathrm{TST}^{\mathrm{b}}$ & 0.78 & 0.017 & 46 \\
\hline Centroid-density QTST ${ }^{\mathrm{b}}$ & 1.1 & 0.026 & 40 \\
\hline $\operatorname{MDQT}^{\mathrm{c}}(1 \mathrm{D}, \text { reversal })^{\mathrm{d}}$ & 7.8 & 2.0 & 3.9 \\
\hline $\operatorname{MDQT}^{\mathrm{e}}(1 \mathrm{D}, \text { no reversal })^{\mathrm{d}}$ & 10 & 2.2 & 4.5 \\
\hline $\operatorname{MDQT}^{\mathrm{e}}(2 \mathrm{D}, \text { no reversal })^{\mathrm{d}}$ & 13 & 2.0 & 6.5 \\
\hline VTST/MT ${ }^{\mathrm{a}}(\mathrm{ES})^{\mathrm{f}}$ & 16 & 1.0 & 16 \\
\hline VTST/MT ${ }^{\mathrm{a}}(\mathrm{NES})^{\mathrm{f}}$ & 13 & 0.85 & 15 \\
\hline Quantum instanton theory ${ }^{\mathrm{g}}$ & 17 & 0.36 & 47 \\
\hline Hansen-Andersen QTST ${ }^{\mathrm{g}}$ & 13 & 0.33 & 39 \\
\hline Centroid density QTST ${ }^{g}$ & 12 & 0.28 & 43 \\
\hline
\end{tabular}

${ }^{\mathrm{a}}$ McRae et al. (Ref. 31).

${ }^{\mathrm{b}}$ Azzouz and Borgis (Ref. 27).

${ }^{\mathrm{c}}$ Hammes-Schiffer and Tully (Ref. 28).

${ }^{d} 1 D$ scheme quantizes the proton only, while $2 \mathrm{D}$ scheme quantizes the proton and the donor-acceptor distance $R_{A B}$. "Reversal" refers to the velocity reversal procedure for classically forbidden transitions in surface hopping algorithm.

${ }^{\mathrm{e}}$ Kim and Hammes-Schiffer (Ref. 29).

f"ES" and "NES" stands for the equilibrium and nonequilibrium solvation models, respectively.

${ }^{\mathrm{g}} \mathrm{Scheme}$ (III) in Table III. 
This magnitude of difference is much smaller than that assumed previously (i.e., two orders of magnitude), thus significantly reconciling different theoretical methodologies used. On the other hand, the uncertainty in the $H / D$ KIE still remains rather large (4-48), due mainly to the large variation in the $D$ transfer rate.

Further tracking down the remaining discrepancy in Table IV is difficult with the present information available, but it is worth noting that the QTST rates are likely to more or less overestimate the true rates of the Azzouz-Borgis model. As is well known, a family of QTST provide their best accuracy when the relevant dynamics is of "direct" nature, i.e., when there is no recrossing of thermal wave packets. The violation of this condition usually leads to an overestimation of the rate due essentially to the neglect of recurrences in the flux-flux correlation function. In fact, the QI theory overestimates the rate constant of the gas-phase collinear $\mathrm{Cl}+\mathrm{HCl}$ reaction at $T=250 \mathrm{~K}$ by a factor of 2 , since the heavy chlorine atoms cannot dissipate the fast hydrogen motions very rapidly. ${ }^{24}$ On the other hand, the error is diminished to as small as $10 \%$ for the $\mathrm{Cl}+\mathrm{DCl}$ reaction because of the reduced difference in the relevant time scales. ${ }^{24}$ Here we recall that the AHB complex in the Azzouz-Borgis problem is a typical collinear "heavy-light-heavy" system; i.e., the Lennard-Jones centers $A$ and $B$ are given unusually large masses of phenol (93 amu) and trimethylamine (59 amu), respectively, while the proton is restrained to move along the $A B$ axis. It is thus possible that this simplified feature of the Azzouz-Borgis model may enhance the dynamical recrossing of the proton than in other realistic hydrogen transfer reactions in condensed phase, although the coupling with the polar solvent should suppress the recrossing effect to some extent. ${ }^{3}$ It is thus highly desirable to quantify the degree of recrossing by using some approximate realtime propagation methods ${ }^{43-49}$ or numerical analytic continuation approaches, ${ }^{50-53}$ but this is clearly beyond the scope of this paper and will be addressed elsewhere.

\section{CONCLUDING REMARKS}

We have demonstrated that it is feasible to apply the QI theory, a recently proposed QTST, to a realistic proton transfer reaction in a condensed phase. The necessary computational effort was rather moderate and slightly greater than that required for the centroid-density QTST, the additional cost arising from the evaluation of the factor $\nu$ in Eq. (2.17). We have also described how the present path integral approach can be used to evaluate the Hansen-Andersen QTST. This is of practical significance because there were no established computational schemes for the latter except for quantum basis set methods, which severely hindered the application of the Hansen-Andersen QTST to realistic chemical reactions. Another key ingredient in this paper is the idea of obtaining the kinetic isotope effect (KIE) directly from thermodynamic integration of the rate with respect to hydrogen mass. This thus avoids repeated calculations of the activation free energy for the $H$ and $D$ transfer and is useful in situations where one's interest is in KIE rather than in the absolute rates, or when it is difficult to evaluate the activation free energy but still the mass integral in Eq. (2.46) can be calculated with tolerable statistical noise using a few-point quadrature over hydrogen mass.

The present application of the QI theory to the AzzouzBorgis problem shows that three different QTSTs (namely, the QI theory, the Hansen-Andersen method, and the centroid-density QTST) give very similar estimates of the rate, within $30 \%$ of each other, and the resulting rates are in reasonable agreement with the results of MDQT and VTST/MT (within an order of magnitude). Further, it was identified that the large discrepancy in the previously calculated rates is partially due to slightly different parametrization of the Azzouz-Borgis model.

Although dynamical recrossing may be nonnegligible in the present work due to the simplicity of the Azzouz-Borgis model, such effects are not of serious concern in more practical applications, because in the latter cases the hydrogen atom moves in full $3 d$ space and this largely diminishes the recrossing effect, ${ }^{54}$ as indicated by numerous simulation studies on enzymatically catalyzed hydrogen transfer reactions. ${ }^{6,55}$ On the other hand, the present path integral based QTST has the clear advantage that all the tunneling contributions are included without bias (i.e., one does not need to specify a tunneling path), and that it is possible to include the quantum effects of proximate heavy atoms with mild increase in CPU time. To see whether these features of the QI theory can indeed be exploited in more practical problems in solution phase and in biological systems, however, needs further theoretical studies.

\section{ACKNOWLEDGMENTS}

This work was supported by the Director, Office of Science, Office of Basic Energy Sciences, Chemical Sciences, Geosciences, and Biosciences Division, U.S. Department of Energy under Contract No. DE AC03-76SF00098 and by the National Science Foundation Grant No. CHE-0096576. We also acknowledge a generous allocation of supercomputing time from the National Energy Research Scientific Computing Center (NERSC).

\section{APPENDIX A: KINETIC ENERGY OF THE H-BONDING COMPLEX}

The solute kinetic energy in Eq. (2.1) is not strictly identical to that employed in previous work, but the difference can be shown to be negligible as follows. First, the kinetic energy used by McRae et al. $^{31}$ and Kim and HammesSchiffer $^{29}$ is of the form

$$
T_{\text {gas }}=\frac{1}{2} M \dot{\mathbf{R}}_{\mathrm{c} . \mathrm{m} .}^{2}+\frac{1}{2}\left(\dot{r} \dot{R}_{A B}\right) \boldsymbol{\mu}\left(\begin{array}{c}
\dot{r} \\
\dot{R}_{A B}
\end{array}\right)+\frac{1}{2} I\left(r, R_{A B}\right) \dot{\boldsymbol{\Omega}}^{2},
$$

where $M=M_{A}+M_{B}+m_{H}, \mathbf{R}_{\mathrm{c} . \mathrm{m} \text {. }}$ is the center-of-mass of the $\mathrm{H}$-bonding complex, $\boldsymbol{\mu}$ is the effective mass tensor,

$$
\boldsymbol{\mu}=\frac{1}{M}\left(\begin{array}{cc}
\left(M_{A}+M_{B}\right) m_{H} & -m_{H} M_{B} \\
-m_{H} M_{B} & \left(M_{A}+m_{H}\right) M_{B}
\end{array}\right),
$$

$I\left(r, R_{A B}\right)$ is the inertia moment, 


$$
I\left(r, R_{A B}\right)=\left(\begin{array}{ll}
r & R_{A B}
\end{array}\right) \mu\left(\begin{array}{c}
r \\
R_{A B}
\end{array}\right),
$$

and $\boldsymbol{\Omega}$ is the orientational unit vector of the complex. Now approximating the mass tensor as

$$
\boldsymbol{\mu} \approx\left(\begin{array}{cc}
m_{H} & 0 \\
0 & \mu_{A B}
\end{array}\right)
$$

and the moment of inertia as $I \approx \mu_{A B} R_{A B}^{2}$ with $\mu_{A B}$ $=M_{A} M_{B} /\left(M_{A}+M_{B}\right)$ based on the fact that $m_{H} \ll M_{A}, M_{B}$, we obtain the following kinetic energy:

$$
T_{\text {gas }}=\frac{1}{2} M \dot{\mathbf{R}}_{\text {c.m. }}^{2}+\frac{1}{2}\left(m_{H} \dot{r}^{2}+\mu_{A B} \dot{R}_{A B}^{2}\right)+\frac{1}{2} \mu_{A B} R_{A B}^{2} \dot{\mathbf{\Omega}}^{2} .
$$

The above form neglects the kinetic coupling between $r$ and $R_{A B}$ and was employed initially by Azzouz and Borgis. ${ }^{27}$ Further neglecting the contribution of the proton to the translational energy of the solute, $1 / 2 M \dot{\mathbf{R}}_{\mathrm{c} . \mathrm{m} \text {. }}^{2}$, and combining all the terms related to the $A$ and $B$ sites, we arrive at the present Cartesian form of the kinetic energy in Eq. (2.1). Although we have also carried out some additional path integral calculations to quantify the effect of the coupling between $r$ and $R_{A B}$, the resulting changes in the rate were at most a few percent and did not affect the main conclusions in this paper.

\section{APPENDIX B: THERMODYNAMIC ESTIMATOR FOR $\ddot{C}_{\mathrm{dd}}(0) / C_{\mathrm{dd}}(0)$}

The thermodynamic estimator for $\ddot{C}_{\mathrm{dd}}(0) / C_{\mathrm{dd}}(0)$ in Eq. (2.49), which is a generalization of Eq. (2.32) to the multislice treatment of the donor-acceptor modes, can be obtained as follows. First, we discretize $C_{\mathrm{dd}}(-i \hbar \lambda)$ as

$$
\begin{aligned}
C_{\mathrm{dd}}(- & i \hbar \lambda) \\
= & \operatorname{tr}\left[e^{-(\beta / 2+\lambda) \hat{H}} \delta\left(\hat{r}-r^{\ddagger}\right) e^{-(\beta / 2-\lambda) \hat{H}} \delta\left(\hat{r}-r^{\ddagger}\right)\right] \\
= & \int d r^{(1)} \ldots \int d r^{(P)} \int d \mathbf{R}^{(1)} \ldots \\
& \times \int d \mathbf{R}^{(P)} \delta\left(r^{(0)}-r^{\ddagger}\right) \delta\left(r^{(P / 2)}-r^{\ddagger}\right) \\
& \times \prod_{s=1}^{P}\left\langle r^{(s)} \mathbf{R}^{(s)}\left|\exp \left(-\Delta \beta^{(s)} \hat{H}\right)\right| r^{(s-1)} \mathbf{R}^{(s-1)}\right\rangle,
\end{aligned}
$$

where $\Delta \beta^{(s)}=\beta / P+c^{(s)} \lambda$ with $c^{(s)}$ being the coefficients given by Eq. (2.33). Using the primitive approximation to the high-temperature Boltzmann operator and applying the grouping procedure for $\mathbf{R}$ in Sec. II F, we have

$$
\begin{aligned}
C_{\mathrm{dd}}(-i \hbar \lambda)= & \int d r^{(1)} \ldots \int d r^{(P)} \int d \mathbf{R}^{[1] \ldots} \\
& \times \int d \mathbf{R}^{\left[P^{\prime}\right]} \delta\left(r^{(0)}-r^{\ddagger}\right) \delta\left(r^{(P / 2)}-r^{\ddagger}\right) \\
& \times\left[\prod_{s=1}^{P}\left\langle r^{(s)}\left|\exp \left(-\Delta \beta^{(s)} \hat{T}_{r}\right)\right| r^{(s-1)}\right\rangle\right]
\end{aligned}
$$

$$
\begin{aligned}
& \times\left[\prod_{\sigma=1}^{P^{\prime}}\left\langle\mathbf{R}^{[\sigma]}\left|\exp \left(-\Delta \beta^{[\sigma]} \hat{T}_{R}\right)\right| \mathbf{R}^{[\sigma-1]}\right\rangle\right] \\
& \times \exp \left\{-\sum_{s=1}^{P} \Delta \beta^{(s)} \bar{V}^{(s)}\right\},
\end{aligned}
$$

where $\bar{V}^{(s)}$ is the average potential in Eq. (2.50), and $\Delta \beta^{[\sigma]}$ is defined by

$$
\Delta \beta^{[\sigma]}=\sum_{s=s_{0}+L(\sigma-1)+1}^{s_{0}+L \sigma} \Delta \beta^{(s)}=\beta / P^{\prime}+C^{[\sigma]} \lambda
$$

with $C^{[\sigma]}$ given by Eq. (2.51). Taking the second derivative of Eq. (B2) with respect to $\lambda$ yields the desired estimator. We note that the coefficient $c^{(s)}$ in Eq. (2.33) is chosen such that it vanishes for time slices $s=1, P / 2, P / 2+1$, and $P$ which neighbor the flux operator in $C_{\mathrm{ff}}(0)$. The independence of these slices from $\lambda$ greatly simplifies the resulting estimator for $\ddot{C}_{\text {ff }}(0) / C_{\text {ff }}(0)$ in Eq. (2.40), since the action of the flux operator and the derivative with respect to $\lambda$ can be performed independently (see Ref. 22 and its Appendix A for related discussions).

${ }^{1}$ A. Kohen, R. Cannio, S. Bartolucci, and J. P. Klinman, Nature (London) 399, 496 (1999); M. J. Knapp and J. P. Klinman, Eur. J. Biochem. 269, 3113 (2002).

${ }^{2}$ J. Basran, S. Patel, M. J. Sutcliffe, and N. S. Scrutton, J. Biol. Chem. 276, 6234 (2001); M. J. Sutcliffe and N. S. Scrutton, Eur. J. Biochem. 269, 3096 (2002).

${ }^{3}$ D. Borgis and J. T. Hynes, J. Chem. Phys. 94, 3619 (1991); J. Phys. Chem. 100, 1118 (1996).

${ }^{4}$ J. Lobaugh and G. A. Voth, J. Chem. Phys. 100, 3039 (1994).

${ }^{5}$ J. Villa and A. Warshel, J. Phys. Chem. B 105, 7887 (2001).

${ }^{6}$ J. Gao and D. G. Truhlar, Annu. Rev. Phys. Chem. 53, 467 (2002).

${ }^{7}$ P. K. Agarwal, S. R. Billeter, P. T. Ravi Rajagopalan, S. J. Benkovic, and S. Hammes-Schiffer, Proc. Natl. Acad. Sci. U.S.A. 99, 2794 (2002); S. Hammes-Schiffer, Curr. Opin. Struct. Biol. 14, 192 (2004).

${ }^{8}$ D. Antoniou and S. D. Schwartz, Proc. Natl. Acad. Sci. U.S.A. 94, 12360 (1997); D. Antoniou, S. Caratzoulas, C. Kalyanaraman, J. S. Mincer, and S. D. Schwartz, Eur. J. Biochem. 269, 3103 (2002).

${ }^{9}$ R. P. Feynman and A. R. Hibbs, Quantum Mechanics and Path Integrals (McGraw-Hill, New York, 1965).

${ }^{10}$ D. M. Ceperley, Rev. Mod. Phys. 67, 279 (1995).

${ }^{11}$ M. J. Gillan, J. Phys. C 20, 3621 (1987); Phys. Rev. Lett. 58, 563 (1987).

${ }^{12}$ G. A. Voth, D. Chandler, and W. H. Miller, J. Chem. Phys. 91, 7749 (1989); J. Phys. Chem. 93, 7009 (1989).

${ }^{13}$ G. A. Voth, J. Phys. Chem. 97, 8365 (1993).

${ }^{14}$ S. Jang and G. A. Voth, J. Chem. Phys. 112, 8747 (2000).

${ }^{15}$ N. F. Hansen and H. C. Andersen, J. Chem. Phys. 101, 6032 (1994); J. Phys. Chem. 100, 1137 (1996).

${ }^{16}$ W. H. Miller, S. D. Schwartz, and J. W. Tromp, J. Chem. Phys. 79, 4889 (1983).

${ }^{17}$ W. H. Thompson, J. Chem. Phys. 110, 4221 (1999).

${ }^{18}$ W. H. Miller, Y. Zhao, M. Ceotto, and S. Yang, J. Chem. Phys. 119, 1329 (2003).

${ }^{19}$ W. H. Miller, J. Chem. Phys. 62, 1899 (1975).

${ }^{20}$ S. Coleman, Phys. Rev. D 15, 2929 (1977).

${ }^{21}$ S. Chapman, B. C. Garrett, and W. H. Miller, J. Chem. Phys. 63, 2710 (1975).

${ }^{22}$ T. Yamamoto and W. H. Miller, J. Chem. Phys. 120, 3086 (2004).

${ }^{23}$ Y. Zhao, T. Yamamoto, and W. H. Miller J. Chem. Phys. 120, 3100 (2004).

${ }^{24}$ M. Ceotto and W. H. Miller, J. Chem. Phys. 120, 6356 (2004).

${ }^{25}$ C. Venkataraman and W. H. Miller, J. Phys. Chem. A 108, 3035 (2004).

${ }^{26}$ M. Ceotto, S. Yang, and W. H. Miller, J. Chem. Phys. (in press).

${ }^{27}$ H. Azzouz and D. Borgis, J. Chem. Phys. 98, 7361 (1993); J. Mol. Liq. 61, 17 (1994); ibid. 63, 89 (1995).

${ }^{28}$ S. Hammes-Schiffer and J. C. Tully, J. Chem. Phys. 101, 4657 (1994).

${ }^{29}$ S. Y. Kim and S. Hammes-Schiffer, J. Chem. Phys. 119, 4389 (2003). 
${ }^{30}$ D. Antoniou and S. D. Schwartz, J. Chem. Phys. 110, 465 (1999); 110, 7359 (1999)

${ }^{31}$ R. P. McRae, G. K. Schenter, B. C. Garrett, Z. Svetlicic, and D. G. Truhlar, J. Chem. Phys. 115, 8460 (2001).

${ }^{32}$ More precisely, Azzouz and Borgis employed an $R_{A B}$-dependent definition of the dividing surface as $r^{\ddagger}=r^{\ddagger}\left(R_{A B}\right)$, but this dependence is rather weak and is thus neglected in this paper. See Appendix B in Ref. 31 for related discussions.

${ }^{33}$ In this paper we do not consider the two dividing-surface version of the QI theory in order to simplify the overall discussion on various approximate rates.

${ }^{34}$ C. Predescu and W. H. Miller, J. Phys. Chem. B (in press).

${ }^{35}$ See, for example, Y. Li and W. H. Miller, Mol. Phys. (to be published).

${ }^{36}$ D. Marx and M. H. Müser, J. Phys.: Condens. Matter 11, R117 (1999).

${ }^{37}$ E. L. Pollock and D. M. Ceperley, Phys. Rev. B 30, 2555 (1984).

${ }^{38}$ M. Sprik, M. L. Klein, and D. Chandler, Phys. Rev. B 31, 4234 (1985).

${ }^{39}$ M. E. Tuckerman, B. J. Berne, G. J. Martyna, and M. L. Klein, J. Chem. Phys. 99, 2796 (1993).

${ }^{40}$ B. Hetenyi, K. Bernacki, and B. J. Berne, J. Chem. Phys. 117, 8203 (2002).

${ }^{41}$ Here we did not perform the adaptive umbrella sampling but instead used a simple Gaussian bias potential, $-V_{0} \exp \left[-\left(r-r^{*}\right)^{2} / 2 \sigma^{2}\right]$ with $V_{0}$ $=3.0-3.5 \mathrm{kcal} / \mathrm{mol}$ and $\sigma=0.1 \AA$, in order to obtain sufficient statistics in the barrier region.

${ }^{42}$ The rates obtained by Antoniou and Schwartz (Ref. 30) using the quantum
Kramers approach are not included here, because their rates vary by more than two orders of magnitude depending on a specific choice of the system-bath Hamiltonian (see Table V in Ref. 31). We conjecture that this sensitivity of the rate may result from the fact that the donor-acceptor modes are treated as a harmonic bath on the same footing with the solvent molecules.

${ }^{43}$ M. Thoss and H. Wang, Annu. Rev. Phys. Chem. 55, 299 (2004).

${ }^{44}$ T. Yamamoto, H. Wang, and W. H. Miller, J. Chem. Phys. 116, 7335 (2002).

${ }^{45}$ E. Jezek and N. Makri, J. Phys. Chem. A 105, 2851 (2001).

${ }^{46}$ N. J. Wright and N. Makri, J. Chem. Phys. 119, 1634 (2003).

${ }^{47}$ E. Geva, Q. Shi, and G. A. Voth, J. Chem. Phys. 115, 9209 (2001).

${ }^{48}$ J. A. Poulsen, G. Nyman, and P. J. Rossky, J. Chem. Phys. 119, 12179 (2003).

${ }^{49}$ I. R. Craig and D. E. Manolopoulos, J. Chem. Phys. 121, 3368 (2004).

${ }^{50}$ K. Yamashita and W. H. Miller, J. Chem. Phys. 82, 5475 (1985).

${ }^{51}$ E. Rabani, G. Krilov, and B. J. Berne, J. Chem. Phys. 112, 2605 (2000).

${ }^{52}$ G. Krilov, E. Sim, and B. J. Berne, J. Chem. Phys. 114, 1075 (2001); E. Sim, G. Krilov, and B. J. Berne, J. Phys. Chem. A 105, 2824 (2001).

${ }^{53}$ B. G. Cheney and H. C. Andersen, J. Chem. Phys. 118, 9542 (2003).

${ }^{54}$ S. Chapman, S. M. Hornstein, and W. H. Miller, J. Am. Chem. Soc. 97, 892 (1975).

${ }^{55}$ M. Garcia-Viloca, J. Gao, M. Karplus, and D. G. Truhlar, Science 303, 186 (2004). 